\title{
Relação do Tamanho de Sementes de Milho e Doses de Fungicida no Controle de Stenocarpella maydis*
}

\author{
Ênia M. Carvalhoํ, José C. Machado ${ }^{1 * *}$, Edila V.R. Von Pinho ${ }^{2}$ Edson A. Pozza ${ }^{1}$ \& Paulo E. R. Prado ${ }^{1}$ \\ ${ }^{1}$ Departamento de Fitopatologia, ${ }^{2}$ Departamento de Agricultura, Universidade Federal de Lavras, CEP 37200-000, \\ Lavras, MG, e-mail: machado@ufla.br
}

(Aceito para publicação em 13/02/2004)

Autor para correspondência: José da Cruz Machado

CARVALHO, M.C., MACHADO, J.C., VON PINHO, R.E.V., POZZA, E.A., \& PRADO, P.E.R. Relação do tamanho da sementes de milho e doses de fungicida no controle de Stenocarpella maydis. Fitopatologia Brasileira 29:389-393. 2004.

\section{RESUMO}

Para o controle integrado de doenças, o tratamento químico das sementes de milho (Zea mays) torna-se indispensável, sendo a sua eficácia e influenciada por vários fatores, entre eles o tamanho das sementes. O objetivo do trabalho foi avaliar a relação do tamanho da semente e doses do fungicida thiabendazole no controle de Stenocarpella maydis, considerando-se diferentes tamanhos de sementes e doses do fungicida. As sementes classificadas nas peneiras 19,22 e 24 foram inoculadas com $S$. maydis e tratadas com o fungicida thiabendazole em três doses, sendo avaliadas a incidência do patógeno e desempenho das sementes por meio de testes de qualidade fisiológica. Constatou-se com o tratamento fungicida, aumento na germinação, maior peso de plântulas e redução de tombamento provocado por $S$. maydis, havendo comportamento diferenciado do tratamento químico em relação ao tamanho de sementes e doses do produto.

Palavras-chave adicionais: Stenocarpella maydis, Zea mays, sementes, thiabendazole.

\section{ABSTRACT}

Relation of maize seed size and doses of fungicide in controlling Stenocarpella maydis

To prevent disease dissemination chemical treatment of maize (Zea mays) seeds becomes indispensable. However, the efficacy of this treatment may be influenced by a number of factors, such as seed size. Thus, the objective of this work was to evaluate the efficacy of the fungicide thiabendazole in treatment of maize seed to control $S$. maydis, taking into account different seed sizes. The seeds classified according to sieves 19, 22 and 24 were inoculated with S. maydis and treated with the fungicide thiabendazole in three doses. To evaluate the effect of seed treatment, test of germination on paper roll substrate, health test, (blotter method), and the dry weight of seedlings were considered. Seed treatment with fungicide provided conditions for increased germination and weight of seedlings, and reduced dampingoff, caused by $S$. maydis. The efficacy of the fungicide seed treatment varied according to seed size and doses of the fungicide used.

\section{INTRODUÇÃO}

A qualidade sanitária e fisiológica das sementes de milho (Zea mays L.) influencia a produtividade desta cultura, em função do papel que desempenham tanto no estabelecimento da lavoura quanto na disseminação de doenças, interferindo, dessa forma, nos índices de produtividade. Entre os fungos transmitidos por essa via, destaca-se Stenocarpella maydis (Berk). Sacc. (sin. Diplodia maydis), pelo potencial de causar danos direto às sementes (Rheeder, et al., 1990; Shurtheff, 1992; Büll \& Cantarella, 1993; Reis \& Casa, 1996; Casa, 1997; Casa et al., 1998) e por estar relacionado com produção de toxinas danosas ao homem e animais. Esse fungo é o único patógeno do milho (potencial) a ser enquadrado na proposição de tolerância em sementes, elaborada para o Brasil (Portaria SDA n ${ }^{\circ}$ 73/97).

O tamanho de semente é um importante fator envolvido na qualidade de sementes de milho (Von Pinho, 1995; Carvalho \& Nakagawa, 2000; Silva, 2000). Sementes maiores originam

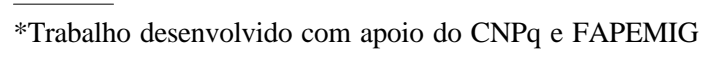

plântulas com maior comprimento e peso de matéria seca (Silva, 2000). Pelo teste de frio este mesmo autor observou que sementes classificadas nas peneiras 20,22 e 24 apresentaram maior porcentagem de germinação em relação às sementes da peneira 18. Silva \& Marcos Filho (1982), estudando a influência do tamanho de sementes de milho em relação à qualidade fisiológica, observaram diferenças apenas no teste de frio, com qualidade superior para as sementes classificadas na peneira 24. No entanto, o tamanho de sementes parece ter maior influência no estabelecimento da cultura; o efeito nessa fase diminui progressivamente com o desenvolvimento vegetativo das plantas (Barnes, 1959).

Um dos métodos para manter as sementes livres de patógenos tem sido o tratamento químico, considerado, como medida de erradicação, uma maneira de contribuir para obtenção de sementes sadias. Entre os diversos fatores que podem interferir na eficácia deste procedimento, incluem-se o tamanho das sementes e doses do produto (Machado, 2000). Em lotes com diferentes tamanhos de sementes, a incorporação de produtos de forma unificada determina comportamento diferencial do tratamento, devido às doses excessivas ou 
reduzidas dos produtos aplicados. $\mathrm{Na}$ maioria dos estudos realizados, a área superficial de contato das sementes em relação às diferentes doses de fungicidas não tem sido considerada. De modo geral, o tratamento de sementes de milho visa principalmente à proteção das mesmas contra os fungos presentes no solo (Pereira, 1986; Casa, 1997), porém, quando o alvo é o controle de $S$. maydis, torna-se uma alternativa viável que contribui para melhorar a emergência das plantas de milho (Pinto, 1992). Casa, et al. (1998) verificaram que o controle de $S$. maydis em milho, por meio de tratamento químico de sementes não proporcionou valores mais elevados de germinação, mas manteve o potencial fisiológico da semente e, reduziu ao mesmo tempo, a transmissão do patógeno.

O tratamento de sementes de milho tem sido realizado com o fungicida captan (Nazareno, 1982; Pereira, 1991; Cicero et al., 1992), no entanto, a eficácia desse produto foi questionável em alguns casos, havendo relatos da interferência do mesmo na emergência de plântulas (Reis et al., 1995). A eficácia de captan no controle de $S$. maydis é melhorada quando aplicado em mistura com thiabendazole, (Casa et al., 1995; Reis et al., 1995; Casa, 1997). O fungicida thiabendazole foi recomendado principalmente para o controle de tombamento, porém, não é indicado para o fungo S. maydis (Andrei, 1999).

Assim, o objetivo deste trabalho foi avaliar a eficácia do tratamento de sementes de milho com o fungicida thiabendazole visando ao controle de $S$. maydis, considerandose diferentes tamanhos de sementes e doses do fungicida.

\section{MATERIAL E MÉTODOS}

O trabalho foi conduzido nos Laboratórios de Patologia de Sementes e de Análise de Sementes dos Departamentos de Fitopatologia e Agricultura da Universidade Federal de Lavras.

Para os ensaios desenvolvidos foram utilizadas sementes de milho, linhagem MTC-0011, oriundas de campo de produção de sementes genéticas da Monsanto, classificadas em grupos, de acordo com as peneiras de crivos circulares, números 19, 22 e 24, sendo as áreas superficiais determinadas pelo Software SIAQS - Sistema Integrado de Análise e Qualidade de Sementes correspondendo a 7200, 6210 e 4380 $\mathrm{m}^{2} / 100 \mathrm{~kg}$, respectivamente.

As sementes foram inoculadas com $S$. maydis pelo método de restrição hídrica (Carvalho, 1999; Costa, 2000; Machado et al., 2001) que consiste no contato das sementes com colônia pura do fungo previamente desenvolvido em meio $\mathrm{BDA}+$ manitol com potencial hídrico ajustado para-1,4 $\mathrm{MPa}$, valor este calculado pelo Software SPPM (Michel \& Radcliffe, 1995). As sementes, com o grau de umidade de $10 \%$ foram tratadas com thiabendazole, nas doses de $15 \mathrm{ml}$ i.a./100 kg de sementes, indicado pelo fabricante e $50 \%$ acima e abaixo deste. Um outro grupo dessas sementes não recebeu tratamento químico consideramdo-se como testemunha.

Para avaliar os efeitos dos tratamentos, cada grupo dos lotes de sementes foi submetido à análise sanitária, pelo método de incubação em papel de filtro desenvolvido por Mario \& Reis (2001), e a análise fisiológica pelos testes de germinação em solo, teste de frio, peso de matéria seca da parte aérea e do sistema radicular de plântulas e Índice de Velocidade de Germinação (IVG). Para os testes de germinação em solo, IVG e peso de plântulas, realizou-se a semeadura em bandejas plásticas $(47 \times 27 \times 08 \mathrm{~cm})$ contendo substrato composto (umidade inicial ajustada para $70 \%$ da capacidade de retenção) de mistura areia e solo de barranco, na proporção $1: 1$, previamente tratado com brometo de metila; após o plantio, as sementes foram cobertas com uma camada $(3 \mathrm{~cm}$ de espessura) do substrato, sendo as bandejas distribuídas aleatoriamente em câmara de crescimento com temperatura ajustada para $25 \pm 2{ }^{\circ} \mathrm{C}$ e fotoperíodo diário de $12 \mathrm{~h}$. Aos sete dias do plantio, determinou-se a porcentagem de plântulas normais, aos 20 dias, a porcentagem de plântulas mortas e o índice de velocidade de germinação pelas contagens diárias das plântulas que apresentavam $1,5 \mathrm{~cm}$ acima do solo, calculado por meio da fórmula desenvolvida por Edmond \& Drapala (1958), citada por Nakagawa (1999). Para o teste de frio, foi adotada a mesma metodologia de semeadura e avaliação descrita para o teste de germinação e IVG, porém, as bandejas, após a semeadura, foram mantidas em câmara fria, a $10^{\circ} \mathrm{C}$ por sete dias e, posteriormente, transferidas para câmara de crescimento nas condições anteriormente descritas. Para avaliar o peso de matéria seca das plântulas desenvolvidas em condições favoráveis para germinação, as sementes foram semeadas em papel "germitest" (papel toalha) umedecido com água destilada esterilizada, 2,5 vezes o peso do papel e incubadas em germinador regulado à temperatura de $25 \pm 2$ ${ }^{\circ} \mathrm{C}$ e fotoperíodo de $12 \mathrm{~h}$. Após sete dias de incubação, avaliouse o peso de matéria seca do sistema radicular e da parte aérea secando-se o material vegetal em estufa com circulação de ar e temperatura ajustada a $65^{\circ} \mathrm{C}$ por três dias.

Para todos os testes realizados, utilizou-se o delineamento experimental inteiramente casualizado, com quatro repetições, em esquema fatorial $3 \times 4$, combinando tamanho de sementes e doses de thiabendazole. As análises estatísticas foram realizadas utilizando-se o programa Sisvar (Ferreira, 2000), sendo realizadas análises de variância (teste de F) e comparação de médias pelo teste $\operatorname{Scott}-\operatorname{Knott}(\mathrm{P} \leq 5 \%)$ ou regressão, de acordo com a natureza qualitativa ou quantitativa, respectivamente.

\section{RESULTADOS E DISCUSSÃO}

O tamanho de sementes afetou, significativamente, a incidência de $S$. maydis e as variáveis fisiológicas que foram analisadas (Tabela 1).

A incidência de $S$. maydis elevou-se significativamente com o tamanho das sementes, e isso decorrido em função da superfície de contato das sementes proporcionada ao micélio fúngico quando da inoculação do patógeno. O tratamento fungicida reduziu significativamente a incidência de $S$. maydis, de aproximadamente $70 \%$ nas sementes não tratadas para menos de $10 \%$ nas sementes tratadas (Figura 1A). A eficácia 
Relação do tamanho de sementes de milho e doses de fungicida...

TABELA 1 - Médias referentes a incidência de Stenocarpella maydis e às variáveis fisiológicas avaliadas em sementes de milho (Zea mays). UFLA, Lavras-MG, 2001

\begin{tabular}{lcccc}
\hline \hline Tamanho & Incidência & \multicolumn{2}{c}{ Teste de Frio } & \multirow{2}{*}{ de semente } \\
\cline { 3 - 5 } de $\boldsymbol{S}$. maydis & Germinação & P.P.A.(g) & \\
\hline Peneira 19 & $17,00 \mathrm{a}$ & $63,13 \mathrm{~b}$ & $0,05 \mathrm{c}$ & $0,03 \mathrm{~b}$ \\
Peneira 22 & $21,88 \mathrm{~b}$ & $69,63 \mathrm{a}$ & $0,09 \mathrm{~b}$ & $0,05 \mathrm{a}$ \\
Peneira 24 & $28,38 \mathrm{c}$ & $61,63 \mathrm{~b}$ & $0,10 \mathrm{a}$ & $0,04 \mathrm{a}$ \\
\hline Média & 22,42 & 64,79 & 0,08 & 0,04 \\
\hline CV (\%) & 16,21 & 12,01 & 18,85 & 16,54 \\
\hline
\end{tabular}

${ }^{1}$ P.P.A. (g): Peso da parte aérea de plântulas

${ }^{2}$ P.S.R. (g): Peso do sistema radicular de plântulas

Médias com a mesma letra não diferem estatisticamente pelo teste ScottKnott $(\mathrm{P} \leq 5 \%)$

de thiabendazole no controle de $S$. maydis foi relatada anteriormente por Reis et al. (1995), Casa et al. (1995) e Casa (1997) em estudos usando esse produto em mistura com o captan.

Quanto aos resultados do teste de germinação em solo sem estresse de temperatura, foi observada significância estatística apenas para o fator dosagem do produto. Quando as sementes foram submetidas à baixa temperatura (teste de frio), observou-se significância para os fatores tamanhos de sementes e dosagem, sendo a maior porcentagem de germinação verificada nas sementes classificadas em peneira 22 (Tabela 1). Em estudos realizados por Barnes (1959) e por Silva (2000), os autores verificaram que sementes maiores, submetidas ao teste de frio foram as que apresentaram maior poder germinativo. Porém no presente trabalho, deve ser ressaltado que as sementes retidas na peneira 24 foram as que apresentaram maior incidência de $S$. maydis, patógeno que pode causar danos irreversíveis às sementes (Rheeder at al., 1990; Casa et al., 1998), o que pode explicar ter verificado também uma baixa germinação, mesmo quando as sementes receberam tratamento químico. Quanto ao fator dose do fungicida, verificou-se aumento na porcentagem de germinação com o aumento das doses utilizadas em ambos os testes (Figuras 1B e 1C).

$\mathrm{O}$ índice de velocidade de germinação das plântulas foi influenciado pela dose do fungicida utilizado (Figura 1D). Sementes tratadas com thiabendazole, na dose intermediária, apresentaram emergência mais rápida.

Em relação ao peso de matéria seca da parte aérea de plântulas, foi observada significância estatística para a interação tamanho de sementes "versus" doses, quando a germinação ocorreu em rolo de papel. Neste teste, para as sementes classificadas na peneira 19 , o peso de matéria seca da parte aérea das plântulas (Figura 2B), não foi influenciado pelas doses do fungicida, o que pode ser entendido, pela relação número de sementes/peso, que nessa amostra houve menor concentração do produto fungicida/semente. Em relação à matéria seca da parte aérea das plântulas provenientes de sementes submetidas à baixa temperatura, observou-se significância para os fatores tamanho de sementes e doses,
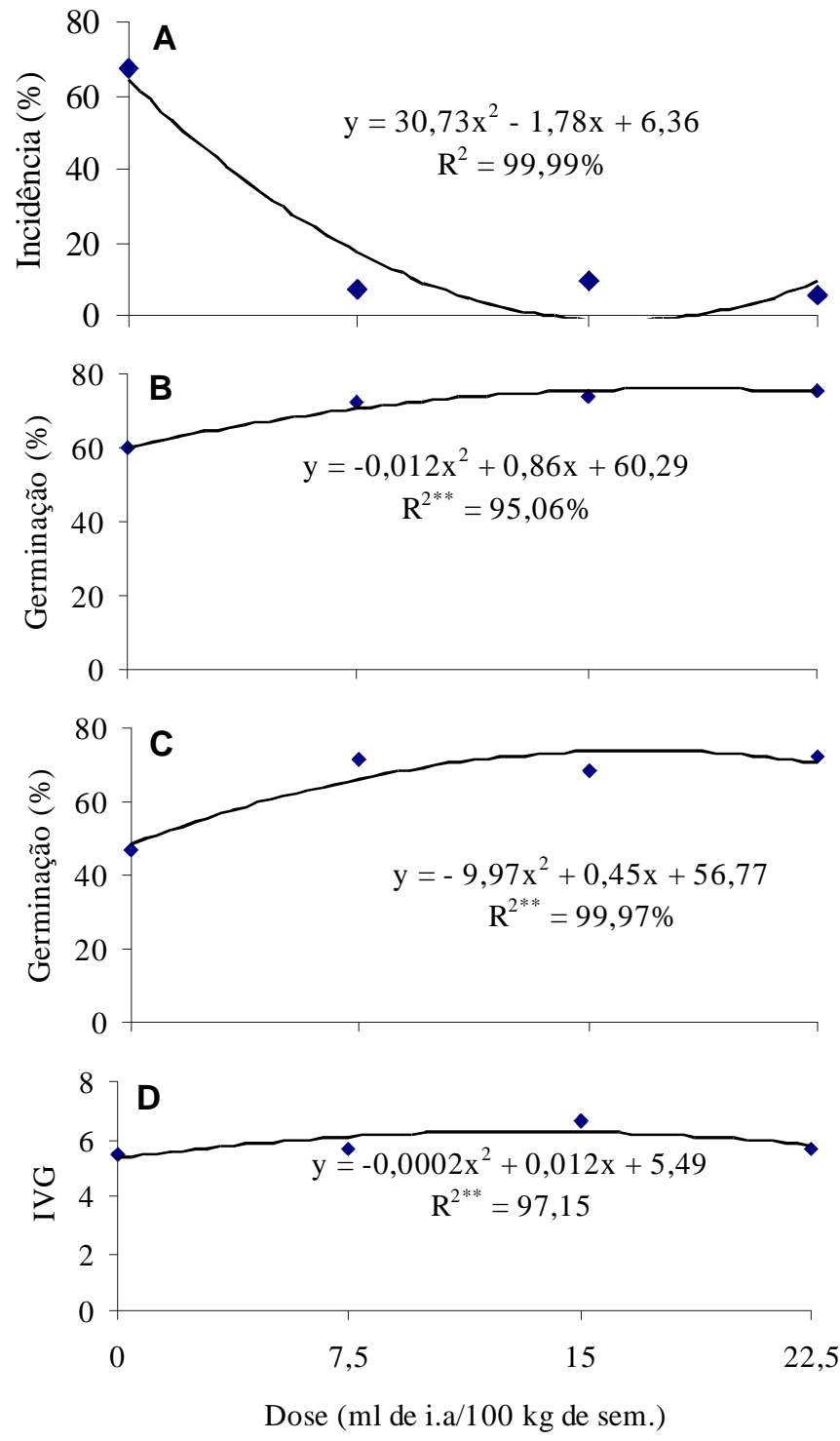

FIG. 1 - Incidência de Stenocarpella maydis (A), germinação (\%) em condições de temperatura ideal (B), e no teste de frio (C) e índice de velocidade de germinação (IVG) (D) de sementes de milho (Zea mays) tratadas com diferentes doses de thiabendazole. UFLA, LavrasMG, 2001.

tendo os valores dessa variável se elevado proporcionalmente ao aumento do tamanho das sementes (Tabela 1). Quanto às doses utilizadas, no teste de frio, foi verificado que sementes tratadas com a dose intermediária originaram plântulas com maior peso da parte aérea, (Figura 2A). Essa mesma tendência foi observada para peso do sistema radicular de plântulas originadas de sementes classificadas em peneira 24, quando submetidas ao teste de frio (Figura 2C). A redução do peso de plântulas com o aumento da dosagem demonstra efeito fitotóxico do fungicida.

Ficou evidenciado que a eficácia do tratamento químico de sementes de milho pode ser influenciada pelo tamanho das sementes, tendo em vista a caracterização de dose em excesso do fungicida utilizado. 
E.M. Carvalho et al.
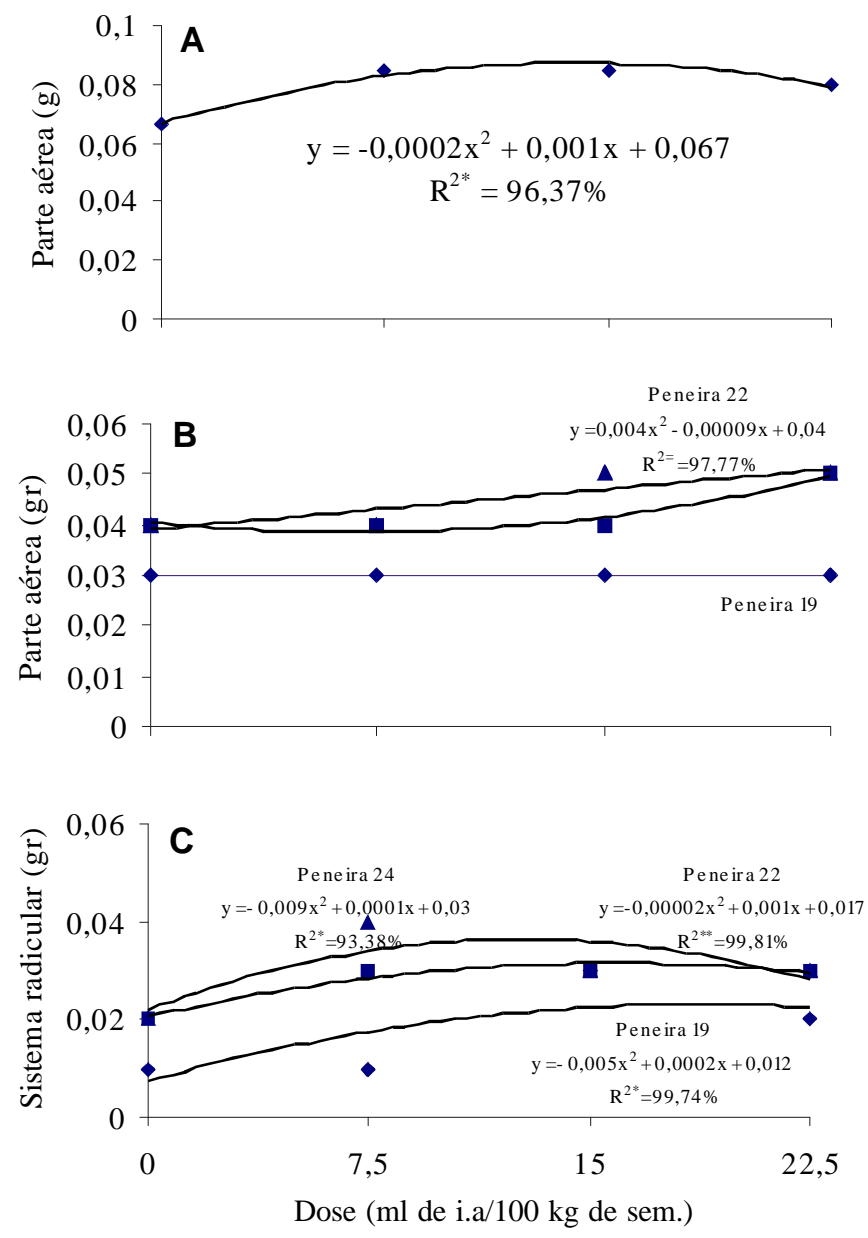

FIG. 2 - Peso de matéria seca da parte aérea de plântulas desenvolvidas de sementes de milho (Zea mays) submetidas ao teste de frio (A) e rolo de papel (B), peso de matéria seca do sistema radicular de plântulas desenvolvidas de sementes submetidas ao teste de frio (C), tratadas com diferentes doses de fungicidas. UFLA-MG, 2001.

\section{REFERÊNCIAS BIBLIOGRÁFICAS}

ANDREI, E. Compêndio de defensivos agrícolas: guia prático de

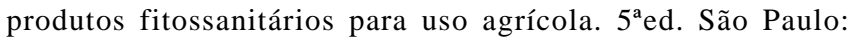
Organização Andrei. 1999.

BARNES, R.F. Seed size has influence on sweet corn matury. Crop and Soils 12:21-22. 1959.

BÜLL, L.T. \& CANTARELLA, H. Cultura do milho: fatores que afetam a produtividade. Piracicaba: POTAFOS. 1993.

CARVALHO, J.C.B. de. Uso da restrição hídrica na inoculação de Colletotrichum lindemunthianum em sementes de feijão (Phaseolus vulgaris L.). (Tese de Mestrado). Lavras. Universidade Federal de Lavras. 1999.

CARVALHO, N.M. \& NAKAGAWA, J. Sementes: ciência, tecnologia e produção. $4^{\mathrm{a} e d .}$ Jaboticabal: Funep. 2000.

CASA, R.T. Diplodia maydis e Diplodia macrospora associados à semente de milho. (Tese de Mestrado). Viçosa. Universidade federal de Viçosa. 1997.

CASA, RT., REIS, E.M., MEDEIROS, C.A. \& MOURA, B. Efeito do tratamento de sementes de milho com fungicidas, na proteção de fungos do solo, no Rio Grande do Sul. Fitopatologia Brasileira 20:633-638. 1995.

CASA, R.T., ZAMBOLIM, L. \& REIS, E.M. Transmissão e controle de diplódia em sementes de milho. Fitopatologia Brasileira 23:436441. 1998.

CICERO, S.M., CHAMMA, H.M.C.P., NOVEMBRE, A.D.L.C. \& MORAES, M.H.D. Physiological and sanitary quality of corn seeds submitted to different fungicide treatments. Seed Science and Technology 20:695-702. 1992.

COSTA, M.L.N. Inoculação de Fusarium oxysporum f. sp. phaseoli em sementes de feijoeiro por meio da restrição hídrica. (Tese de Mestrado). Lavras. Universidade Federal de Lavras. 2000.

FERREIRA, D.F. Análises estatísticas por meio do SISVAR para Windows versão 4.0. Programa e Resumos, Reunião Anual da Região Brasileira da Sociedade Internacional de Biometria, São Carlos, SP. 2000.

MACHADO, J.C. Tratamento de sementes no controle de doenças. Lavras: LAPS/UFLA/FAEPE. 2000.

MACHADO, J.C., CARVALHO, J.C.B., VIEIRA. M.G.G.C. \& GUIMARÃES, R.M. Methodology for infecting seeds by fungi using water restriction technique. Abstract, 26 International Seed Testing Congress-seed Symposium. Angers, France. 2001.

MARIO, J.L. \& REIS, E.M. Método simples para diferenciar Diplodia macrospora de D. Maydis em testes de patologia de sementes de milho. Fitopatologia Brasileira 26:670-672. 2001.

MICHEL, B.E. \& RADCLIFFE, D. A computer program relating solute potencial to solution composition for five solutes. Agronomy Journal 87. 1995.

NAKAGAWA, J. Teste de vigor baseado no desempenho das plântulas. In: Krzyanowski, F.C., Vieira, R.D. \& França Neto, J.B. (Eds.) Vigor de sementes: conceitos e testes. Londrina, Associação Brasileira de Tecnologista de Sementes. 1999. pp.2.1-2.24.

NAZARENO, N.R.X. Controle de doenças. In: Instituto Agronômico Do Paraná. O Milho no Paraná. Londrina, PR. IAPAR. Circular, 29, 1982. pp.149-163.

PEREIRA, O.A.P. Tratamento de sementes de milho no Brasil. In: Mentem, J.O.P. (Ed.). Patógenos em sementes: detecção, danos e controle químico. Piracicaba: ESALQ/FEALQ. 1991. pp.271-279.

PEREIRA, O.A.P. Tratamento de sementes de milho. $2^{\circ}$ Simpósio Brasileiro de Patologia de Sementes, Campinas, SP. 1986. pp.145148.

PINTO, N.F.J.A. Tratamento de sementes de milho com fungicidas visando o controle de Diplodia maydis. Resumos, Fitopatologia Brasileira 17:168. 1992. (Resumo)

REIS, A.C. \& CASA, R.T. Manual de identificação e controle de doenças de milho. Passo Fundo: Aldeia Norte. 1996.

REIS, A.C., REIS, E.M., CASA, R.T. \& FORCELINI, C.A. Erradicação de fungos patogênicos associados a sementes de milho e proteção contra Pythium sp. presente no solo pelo tratamento com fungicidas. Fitopatologia Brasileira 20:585-590. 1995.

RHEEDER, J.P., MARASAS, W.F.O. \& VAN WYK, P.S. Fungal association in corn kernels and effects on germination. Phytopathology 80:131-134. 1990.

SHURTLEFF, A.R. Compendium of corn disease. St. Paul: American Phytopathological Society, 1992. 
Relação do tamanho de sementes de milho e doses de fungicida...

SILVA, S.C. Relação entre o tamanho das sementes de milho (Zea mays L.) com a germinação, o vigor e os componentes da produção de grãos. (Tese de Mestrado). Jaboticabal. Universidade do Estado de São Paulo. 2000.

SILVA, W.R. \& MARCOS FILHO, J. Influência do peso e do tamanho das sementes e milho sobre o desempenho no campo. Pesquisa Agropecuária Brasileira 17:1743-1750. 1982.

VON PINHO, E.V.R. Efeito do tratamento químico sobre a qualidade sanitária e fisiológica de sementes de milho (Zea mays L.) Revista Brasileira de sementes 17:23-28. 1995. 